EPJ manuscript No.

(will be inserted by the editor)

\title{
A Unified Framework for the Pareto Law and Matthew Effect using Scale-Free Networks
}

\author{
Mao-Bin $\mathrm{Hu}^{1}{ }^{\mathrm{a}}{ }^{\text {, }}$ Wen-Xu Wang ${ }^{2}$, Rui Jiang ${ }^{1}$, Qing-Song $\mathrm{Wu}^{1}{ }^{\mathrm{b}}{ }^{\mathrm{b}}$ Bing-Hong Wang ${ }^{2}$, Yong-Hong $\mathrm{Wu}^{3}$ \\ 1. School of Engineering Science, University of Science and Technology of China, Hefei, 230026, P.R.China \\ 2. Nonlinear Science Center and Department of Modern Physics, University of Science and Technology of China, Hefei, 230026, \\ P.R.China \\ 3. Department of Mathematics and Statistics, Curtin University of Technology, Perth, WA6845, Australia
}

Received: date / Revised version: date

\begin{abstract}
We investigate the accumulated wealth distribution by adopting evolutionary games taking place on scale-free networks. The system self-organizes to a critical Pareto distribution (1897) of wealth $P(m) \sim m^{-(v+1)}$ with $1.6<v<2.0$ (which is in agreement with that of U.S. or Japan). Particularly, the agent's personal wealth is proportional to its number of contacts (connectivity), and this leads to the phenomenon that the rich gets richer and the poor gets relatively poorer, which is consistent with the Matthew Effect present in society, economy, science and so on. Though our model is simple, it provides a good representation of cooperation and profit accumulation behavior in economy, and it combines the network theory with econophysics.
\end{abstract}

PACS. 87.23.Ge Dynamics of social systems - 89.75.Hc Networks and genealogical trees - 05.10.-a Computational methods in statistical physics and nonlinear dynamics - 89.75.-k Complex systems

\section{Introduction}

The interaction of many cooperatively interacting agents in economy has many features in common with the statistical physics of interacting systems. A century ago, Pareto (1897) showed that the probability distribution $P(W)$ for income or wealth of an individual in the market decreased , with the wealth $W$ according to a power law [1:

$$
P(W) \propto W^{-(1+v)}
$$

where the value of $v$ was found to lie between 1 and 2 2, 3, 4,5. 5. Studies on real data show that the high-income group indeed follows the Pareto law, with $v$ varying from 1.6 for USA [2] to 1.8-2.2 in Japan [3].

The previous studies of wealth distribution often adopt an ideal-gas model in which each agent is represented by a gas molecule and each trading is a money-conserving collision 6, 7, 8, 9, 10,11]. The model considers a closed economic system where the total money is conserved and the number of economic agents is fixed. Money and average money per agent are equivalent to the energy and temperature in an equilibrium system. Basically, this idealgas model can only reproduce the Gibb distribution or Gaussian-like stationary distribution of money [6]. However, Chatterjee et al introduce the quenched saving propensity of the agents, and the system self-organizes to the

\footnotetext{
a E-mail: humaobin@ustc.edu.cn

b E-mail: qswu@ustc.edu.cn
}

Pareto distribution of money with $v \sim 1$ [10]. We also note that the model is not suitable for studying the material wealth distribution because, in general, the total material wealth of the system will increase with time [7]10].

The unique feature of our work is that we adopt the scale-free network to represent the cooperative structure in population and study the wealth increment by using evolutionary games as a paradigm for economic activities.

A wide range of systems in nature and society can be described as complex networks. Since the discovery of small-world phenomena by Watts and Strogatz [12] and Scale-free phenomena by Barabási and Albert 13 , investigation of complex networks has attracted continuous attention from the physics community [14].

Network theory provides a natural framework to describe the population structure by representing the agents of a given population with the network vertices, and the contacts between those agents with edges [15]. One can easily conclude that well-mixed populations can be represented by complete (fully-connected, regular) networks. Spatially-structured populations are associated with regular networks, exhibiting a degree distribution $d(k)$ which is sharply peaked at a single value of the connectivity $k$, since all agents generally have the same averaged connectivity. Recently, much empirical evidence of real-world social networks has revealed that they are associated with a scale-free, power-law degree distribution, $d(k) \sim k^{-\gamma}$ with $2 \leq \gamma \leq 3$ [14 15 16]. That is, interactions in real-world 
Please give a shorter version with: \authorrunning and \titlerunning prior to \maketitle

networks are heterogeneous that different individuals have different numbers of average neighbors whom they interact with. Thus, the classic regular or random networks are not good representations of many real social networks which likely possess the self-organized mechanism. Hence, in this paper, we adopt the scale-free network model to construct the cooperation structure in population.

The evolutionary game theory has been considered to be an important approach for characterizing and understanding the cooperative behavior in systems consisting of selfish individuals 18 19. Since their introduction, the Prisoner's Dilemma (PD) and the Snowdrift Game (SG) have drawn much attention from scientific communities 20, 21, 22, 23, 24. In both games, two players simultaneously decide whether to cooperate (C) or defect (D). Each player will get a payoff based on his and his opponent's strategy in each step and then the players will choose to change their strategy or to keep their strategy unchanged based on some take-over strategies. One can see that both games' dynamics are very similar to the cooperation and payoff activities between agents in economy and so they are intrinsically suitable for characterizing the payoff and wealth accumulating behavior in populations.

In this paper, we investigate the wealth accumulation of agents playing evolutionary games on the scale-free network. The simulation results show the Pareto wealth distributions along with some remarkable phenomena including the total wealth variation with game parameters, and the Matthew Effect in economy, science, fame, and so on $25,26,27,28$.

\section{Model}

In this paper, the simulation starts from establishing the underlying cooperation network structure according to the most general Barabási-Albert (BA) scale-free network model 13. In this model, starting from $m_{0}$ fully connected vertices, one vertex with $m \leq m_{0}$ edges is attached at each time step in such a way that the probability $\Pi_{i}$ of being connected to the existing vertex $i$ is proportional to the degree $k_{i}$ of the vertex, i.e. $\Pi_{i}=\frac{k_{i}}{\Sigma_{j} k_{j}}$, where $j$ runs over all existing vertices. Initially, an equal percentage of cooperators or defectors was randomly distributed among the agents (vertices) of the population. At each time step, the agents play the PD or SG with their neighbours and get payoff according to the games' payoff matrix.

In the Prisoner's Dilemma, each player can either 'cooperate' (invest in a common good) or 'defect' (exploit the others investment). Two players both receive $R$ upon mutual cooperation and $P$ upon mutual defection. A defector exploiting a cooperator gets an amount $T$ and the exploited cooperator receives $S$, such that $T>R>P>S$. So, 'defect' is the best response to any action by the opponent [24. Thus in a single play of the game, each player should defect. In the Snowdrift Game (SG), the order of $P$ and $S$ is exchanged, such that $T>R>S>P$. Comparing with $\mathrm{PD}, \mathrm{SG}$ is more in favor of cooperation. Following common practice 20.23, we firstly rescale the games such

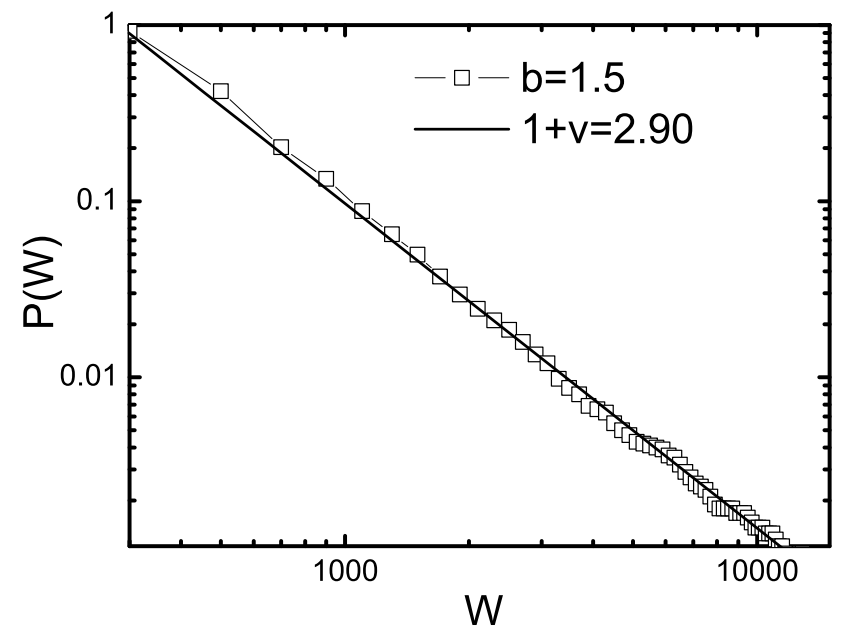

Fig. 1. Wealth distribution $P(W)$ for $N=10^{4}$ agents playing PD game with $b=1.5$ for $10^{5}$ steps. The frequency of cooperators is 0.2137 , and the maximum personal wealth is about 10000 .

that each depends on a single parameter. For the PD, we choose the payoffs to have the values $T=b>1, R=1$, and $P=S=0$, where $1<b \leq 2$ represents the advantage of defectors over cooperators. That is, mutual cooperators each gets 1 , mutual defectors 0 , and $\mathrm{D}$ gets $b$ against $\mathrm{C}$. The parameter $b$ is the only parameter. For the SG, we make $T=1+\beta, R=1, S=1-\beta$, and $P=0$ with $0<\beta<1$ as the only parameter.

Evolution is carried out by implementing the finite population analogue of replicator dynamics [18,23. In each step, all pairs of directly connected individual $x$ and $y$ engage in a single round of a given game. The total payoff of agent $i$ for the step is stored as $P_{i}$. And the accumulative payoff (Wealth) of agent $i$ since the beginning of simulation is stored as $W_{i}$. Then the strategy of each agent (Cooperate or Defect) is updated in parallel according to the following rule: whenever a site $x$ is updated, a neighbor $y$ is drawn at random among all $k_{x}$ neighbors, and the chosen neighbor takes over site $x$ with probability:

$$
P_{x y}=\frac{1}{1+e^{\left(P_{x}-P_{y}\right) / \gamma}},
$$

where $\gamma$ characterizes noise introduced to permit irrational choices [29,30,31], and we make $\gamma=0.1$ as in 30,31.

\section{Simulation Results}

We carry out the simulation for a population of $N=10^{4}$ agents occupying the vertices of a BA scale-free network. The distributions of wealth, total wealth, and k-wealth relation were obtained after a time period of $T=10^{5}$ steps.

We first examine the wealth distribution $P(W)$ of the system. Fig. 1] and Fig. 2 show the $P(W)$ for $\mathrm{PD}(b=1.5)$ and SG $(\beta=0.5)$ respectively. One can see that both 


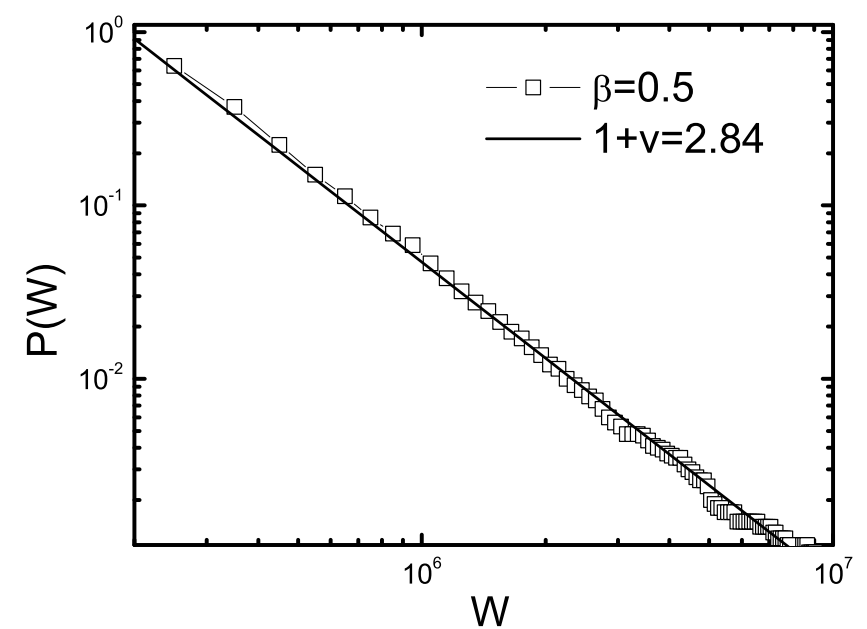

Fig. 2. Wealth distribution $P(W)$ for $N=10^{4}$ agents playing SG game with $\beta=0.5$ for $10^{5}$ steps. The frequency of cooperators is 0.9999 , and the maximum personal wealth is about $10^{7}$.

charts show power-law distribution of personal wealth which is in agreement with Pareto's law with $v=1.90$ and $v=1.84$ respectively. We perform different simulations by altering the values of $b$ and $\beta$, and the results show similar wealth distributions with extremely robust power law. For different simulations, the exponential factor $v$ varies between 1.6 and 2.0 that are in agreement with the empirical values observed in economies including that of U.S (1.60) [2] and Japan $(1.80 \sim 2.20)[\underline{3}$. We focus on the payoff and wealth accumulating behavior in population. In this sense, the wealth distribution we study here essentially corresponds to 'real wealth' or 'material wealth', and not the 'paper money' that is generally conserved in the economic system. We also note that the wealth distribution is independent of the system size $N$ or the simulation time $T$. Although the system's maximum personal wealth is different for Fig. 1 and Fig. 2 because of the difference in cooperators' frequency, the power law persists for both high and low cooperator's frequency cases. All these factors indicate the robustness of our model to reproduce the Pareto Law of economy.

Now we consider the system's total wealth variation with the parameter $b$ or $\beta$. Fig. 3 and Fig. 4 show the variation of total wealth of a $N=10^{4}$ agents system playing PD and SG respectively. One can see from Fig. [3 that the total wealth takes a high value $\left(\approx 4 \times 10^{9}\right)$ when $b$ is relatively small $(\leq 1.10)$. Then there is a bistable region $(1.12<b<1.40)$ where the total wealth can be either high $\left(\approx 4 \times 10^{9}\right)$ or low $\left(\approx 5 \times 10^{5}\right)$. When $b$ is greater than 1.40 , the total wealth remains low $\left(\approx 5 \times 10^{5}\right)$. The high value of the system's total wealth can be as large as $10^{4}$ times of the low value. We note that the total wealth value is related to the frequency of cooperators such that the system's total wealth is high when the frequency is high, and a low total wealth shows up when the frequency is low. For instance, the frequency of cooperators is 0.9999 and

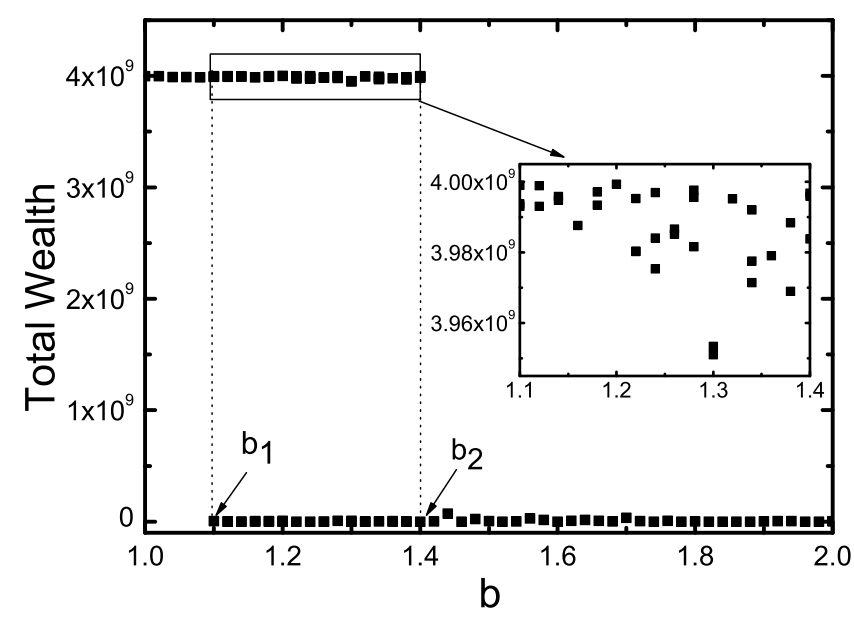

Fig. 3. Total Wealth variation for $N=10^{4}$ agents playing PD game. The arrows with $b_{1}=1.10$ and $b_{2}=1.40$ show the boundaries of the bistable region. The insert shows the fluctuation of the total wealth in the high branch of the bistable region.

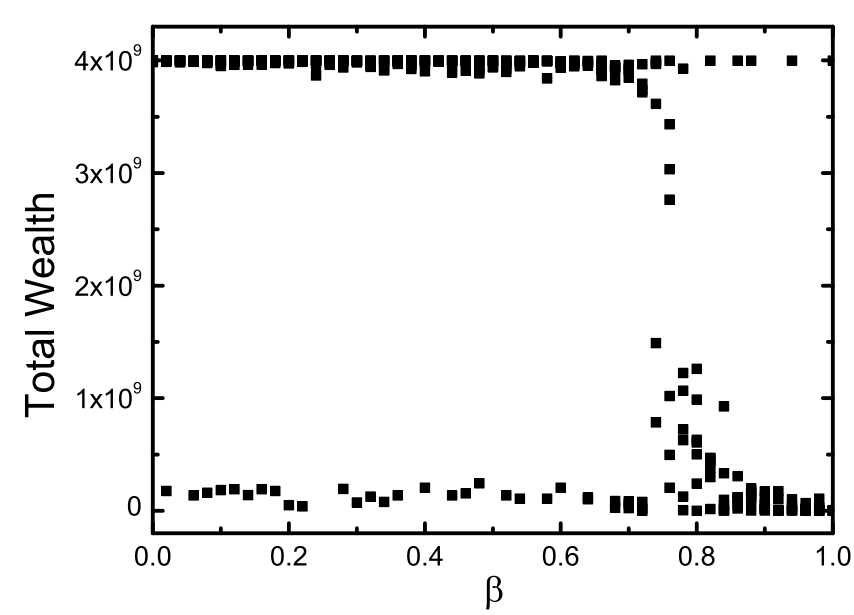

Fig. 4. Total Wealth variation for $N=10^{4}$ agents playing $\mathrm{SG}$ game.

the maximum total wealth is 3996720318 when $b=1.0$. However, the frequency of cooperators is only 0.2137 and the total wealth is only 5461747 when $b=1.5$. This phenomenon implies that when the advantage of defectors over cooperators is too high, the system will take the risk of sharply reducing its total wealth. Thus, a defectorfavored economic rule can prohibit the emergence of cooperators and, what is more, greatly reduce the total wealth of the system.

However, because the SG payoff matrix $T>R>S>$ $P$ is intrinsically cooperator-favored, the total wealth for SG fluctuates as the $\beta$ value changes as shown in Fig. [4

Fig. 5 and Fig. 6 show the relation of personal wealth $W$ with its connectivity $k$. One can see in both cases (PD and SG) that the personal wealth is proportional to its connectivity. Since the number of agents it contacts re- 


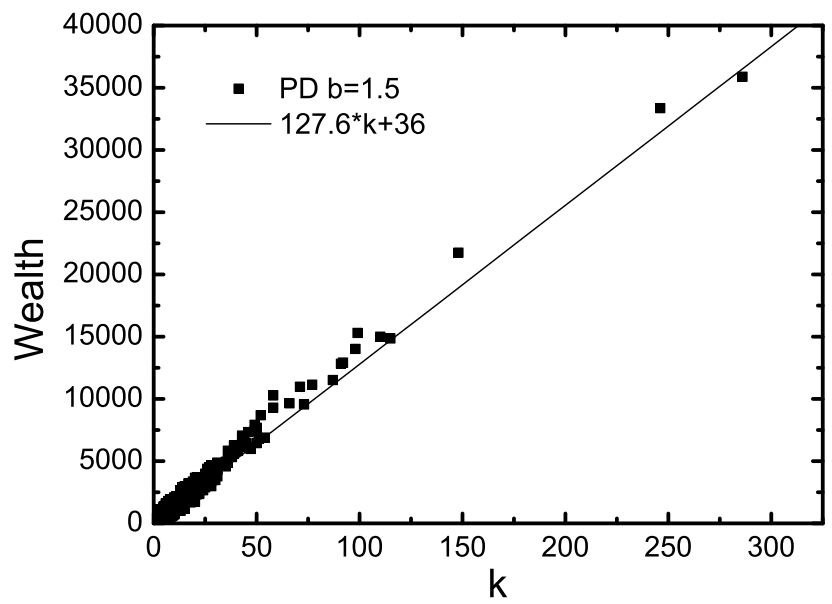

Fig. 5. K-Wealth relation for $N=10^{4}$ agents playing $\mathrm{PD}$ game with $b=1.5$.

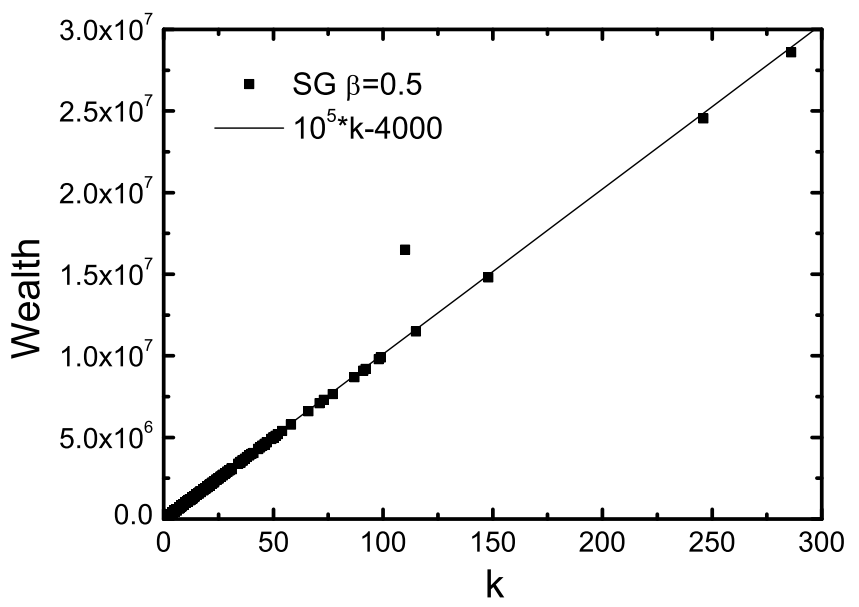

Fig. 6. K-Wealth relation for $N=10^{4}$ agents playing SG game with $\beta=0.5$.

flects the information resources it has, this model also provides a framework to explain the fact that agents with more information resources can gain more profit in modern society's economy.

This proportional relation between personal wealth and its connectivity is also a possible mechanism for the emergence of the Matthew Effect in economy. The "Matthew Effect" refers to the idea that in some areas of life (wealth, achievement, fame, success et al), the rich gets richer and the poor gets poorer [25, 26, 27, 28]. The eminent sociologist Robert Merton used the term "Matthew effect" to describe the deplorable practice of scientists giving exclusive credit to the most distinguished one among several equally deserving candidates [25]. The Matthew effect for Countries (MEC) was also discovered [26]. Our simulations capture a possible underlying mechanism for these phenomena. In Fig. 7 and Fig. 8] the wealth variations of two individual agents are compared. One can see that with both PD and SG, the wealth of the agent with more connectivity exceeds the agent with less connectivity. We

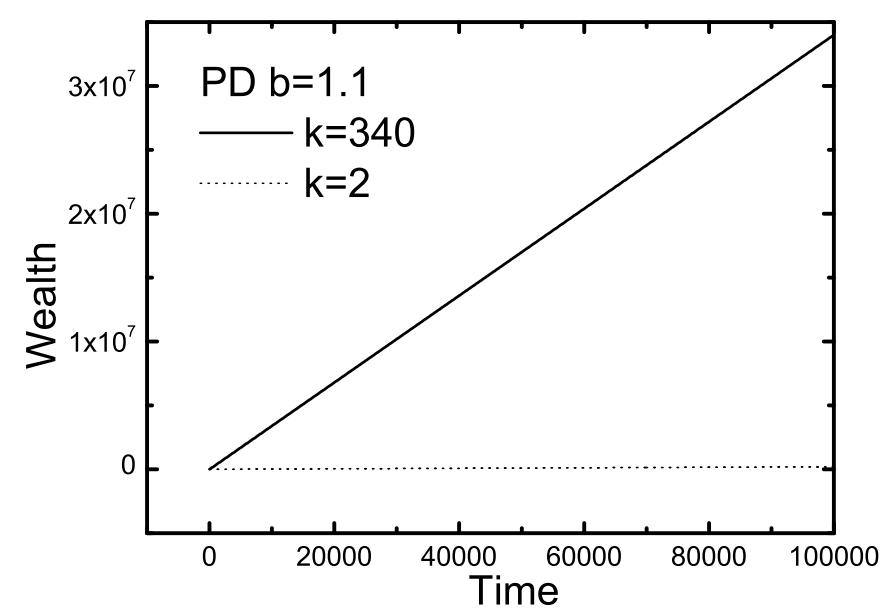

Fig. 7. Matthew Effect in PD game. The one with more connectivity surpass the one with fewer connectivity in their personal wealth.

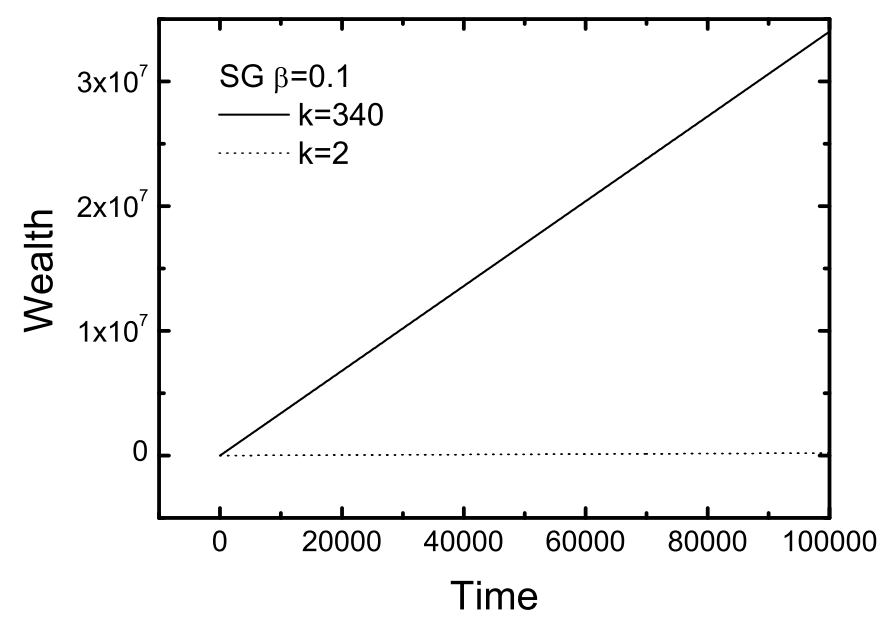

Fig. 8. Matthew Effect in SG game.

note that this tendency remains the same when different values of parameter $b$ or $\beta$ are used. And also the tendency is independent of the system size $N$ or the simulation time $T$. Thus, the agents with more cooperation partners will get richer and richer while those with fewer partners will get relatively poorer. It is true, from our experience, that a successful people (company, country etc) usually have more partners than a unsuccessful one, and this huge relation network will provide him more profits. So, to some extent, our model explains the Matthew Effect from a statistical point of view.

\section{Conclusions}

In conclusion, we have studied the wealth distribution in economy by calculating the accumulative payoff of agents 
involving in revolutionary games on the cooperation network with scale-free property. The simulations confirm Pareto's power law of wealth distribution. And the values of exponential factor $v$ are in agreement with the empirical observations.

The simulation shows that the system's total wealth varies with the game parameters. The results of the $\mathrm{PD}$ game shows that agents tend to cooperate with a frequency of nearly 1.0 and a high total wealth can be achieved when the advantage of defector over cooperator $(b)$ is relatively low. But the total wealth will drop to a very low value when $b$ is high. The total wealth of SG fluctuates as the $\beta$ value changes.

The model also provides a possible explanation for the Matthew Effect from a statistical physics point of view. The simulations show that the agents' personal wealth is proportional to the number of its contacts (connectivity). This leads to the phenomenon that the rich gets richer and the poor gets poorer (Matthew Effect). Thus, in this sense, one has to increase the number of partners in order to gain more profit in modern society. This also suggests a framework to explain why agents with more information resources can gain more profit in modern society's economy, since the connectivity is a representation of an agent's information resource.

It is evident from the above discussions that, our model provides a simple but good approach to study the wealth phenomena in economy, and therefore is worthy of more attention.

\section{ACKNOWLEDGEMENTS}

This work is financially supported by the National Natural Science Foundation of China (Grant No. 10532060, 10404025) and the Australian Research Council through a Discovery Project Grant.

\section{References}

1. V. Pareto, Le Cours d'Economique Politique, Macmillan, Lausanne, Paris (1987).

2. A.A. Dragulescu, V.M. Yakovenko, Physica A 299, 213(2001)

3. S. Moss de Oliveira, P.M.C. de Oliveira, D. Stauer, Evolution, Money, War and Computers, B.G. Tuebner, Stuttgart, Leipzig, (1999).

4. Y. Fujiwara, W. Souma, H. Aoyama, T. Kaizoji, M. Aoki, Physica A 321, 598(2003).

5. M. Levy, S. Solomon, Physica A 242, 90(1997).

6. A. Chakraborti, B.K. Chakrabarti, Eur. P. J. B 17, 167(2000).

7. A. Dragulascu, V.M. Yakovenko, Eur. P. J. B 17, 723(2000).

8. R. Fischer, D. Braun, Physica A 321, 605(2003).

9. Y. Wang, N. Ding, L. Zhang, Physica A 324, 665(2003).

10. A. Chatterjee, B. K. Chakrabarti, S.S. Manna, Physica A 335, 155(2004).

11. N. Xi, N. Ding, Y. Wang, Physica A 357, 543(2005).

12. D.J. Watts, S.H. Strogatz, Nature 393, 440(1998).
13. A.L. Barabási, R. Albert, Science 286, 509(1999).

14. R. Albert, A.L. Barabási, Rev. Mod. Phys. 74, 47(2002).

15. F.C. Santos, J.M. Pacheco, Phys. Rev. Lett. 95, 098104(2005).

16. S.N. Dorogotsev, J.F.F. Mendes, Evolution of Networks: From Biological Nets to the Internet and WWW, Oxford University, Oxford (2003).

17. M.E.J. Newman, Phys. Rev. E 64, 016132(2001).

18. H. Gintis, Game Theory Evolving, Princeton University, Princeton, NJ(2000).

19. A.M. Colman, Game Theory and its Applications in the Social and Biological Sciences, Butterworth-Heinemann, Oxford (1995).

20. M. Nowak, K. Sigmund, Nature (London) 355, 250(1992).

21. M. Nowak, K. Sigmund, Nature (London) 364, 1(1993).

22. M. Nowak, R.M. May, Nature (London) 359, 826(1992); Int. J. Bifurcation Chaos Appl. Sci. Eng. 3, 35(1993).

23. C. Hauert, M. Doebeli, Nature 428, 643(2004).

24. J.M. McNamara, Z. Barta, A.I. Houston, Nature 428, $745(2004)$.

25. R.K. Merton, Science 159,56(1968); ISIS 79, 606(1988).

26. M. Bonitz, E. Bruckner, A. Scharnhorst, Scientometrics 40(3), 407(1997); M. Bonitz, Scientometrics 64(3), $375(2005)$.

27. D.F. Brewer, Physics Today 44(10), 154(1991);

28. R.H. Wade, Inter. J. Health Services 35(4), 631(2005).

29. G. Szabó, C. Töke, Phys. Rev. E 58, 69(1998).

30. G. Szabó, C. Hauert, Phys. Rev. Lett. 89(11), 118101(2002).

31. G. Szabó, J. Vukov, Phys. Rev. E 69, 036107(2004). 